\title{
Profile of Daprodustat in the Treatment of Renal Anemia Due to Chronic Kidney Disease
}

This article was published in the following Dove Press journal:

Therapeutics and Clinical Risk Management

\author{
Taisuke Ishii (1D \\ Tetsuhiro Tanaka \\ Masaomi Nangaku \\ Division of Nephrology and \\ Endocrinology, The University of Tokyo \\ Graduate School of Medicine, Tokyo, \\ Japan
}

\begin{abstract}
Anemia is a major complication of chronic kidney disease (CKD), which mainly results from appropriate erythropoietin production impairment. Prolyl hydroxylase domain (PHD) inhibitors are currently being developed and approved in some countries as a new treatment for CKD patients with anemia due to the stabilization of intracellular hypoxiainducible factor (HIF) $1 \alpha$ and HIF $2 \alpha$ by PHD inhibition. Daprodustat is one of the orally administrated small-molecule HIF-PH inhibitors, leading to an increase in erythropoietin production, which is regulated by HIF. Also, daprodustat is expected to improve iron metabolism. Recently, several clinical trials showed its efficacy and safety in both hemodialysis- and non-hemodialysis- dependent CKD patients. In addition, some international Phase 3 studies are underway to confirm these effects and reveal the safety profile. This article summarizes the development process and results of each clinical trial.
\end{abstract}

Keywords: prolyl hydroxylase domain, hypoxia-inducible factor, erythropoietin, iron

\section{Introduction}

The number of chronic kidney disease (CKD) patients is increasing, estimated to be about 700 million people worldwide. ${ }^{1}$ CKD causes several comorbidities and contributes to worsening quality of life and short life expectancy. One of its comorbidity is anemia, which is caused by several factors, including an insufficient amount of erythropoietin (EPO) production. $^{2}$ In addition, CKD patients with anemia have a high risk for cardiovascular events and death. ${ }^{3}$ Erythropoiesisstimulating agents (ESAs), including recombinant human erythropoietin (rhEPO), can improve renal anemia and quality of life and reduce the need for blood transfusions. $^{4-6}$ However, several studies have revealed that ESA treatment increases cardiovascular events, stroke, and death when the target hemoglobin level is $\geq 13 \mathrm{~g} / \mathrm{dl}^{4,7-9}$ and that high-dose ESA is associated with a cardiovascular risk increase. ${ }^{10}$ Moreover, another major concern is hyporesponsiveness to ESA therapy, which is primarily caused by inflammation and iron deficiency. ${ }^{11}$

Hypoxia-inducible factor prolyl hydroxylase (HIF-PH) inhibitors are a new emerging treatment for renal anemia. ${ }^{12}$ HIF-PH inhibitors maintain hypoxia-inducible factor (HIF) $1 \alpha$ and HIF2 $\alpha$ activity by impeding intracellular HIF degradation, leading to the activation of several HIF-regulated genes, including EPO and iron absorptionand utilization-associated genes. ${ }^{13}$ Previously, international phase 3 clinical trials, which evaluated the efficacy and safety of roxadustat, one of the HIF-PH inhibitors, were reported, ${ }^{14,15}$ and roxadustat was approved in China, Japan, and European countries. One of the orally available, small-molecule HIF-PH inhibitors, which are
Correspondence: Masaomi Nangaku Division of Nephrology and Endocrinology, The University of Tokyo Graduate School of Medicine, 7-3-I Hongo, Bunkyo-ku, Tokyo, I I 38655, Japan Tel +8I-3-38I5-54II

$\mathrm{Fax}+81-3-5800-9826$

Email mnangaku-tky@umin.ac.jp
Therapeutics and Clinical Risk Management 2021:17 155-163

your manuscript

DovePress in $\square$ 
recently investigated, is daprodustat. Several Phase 2 studies showed that it could improve anemia in hemodialysis patients as well as non-hemodialysis-dependent CKD patients and iron utilization. Recently, phase 3 studies are ongoing, with some results being published. Based on these results, daprodustat was approved for the treatment of renal anemia in Japan. ${ }^{16}$ In this review, we summarize the efficacy and safety of daprodustat, which were reported by several phase 2 and phase 3 studies.

\section{Daprodustat Pharmacological Summary}

Daprodustat (GSK1278863) is one of the HIF-PH inhibitors that result in intracellular HIF $1 \alpha$ and HIF $2 \alpha$ stabilization, developed by GlaxoSmithKline. It inhibits prolyl hydroxylase domain (PHD) 1-3 with at least 1000-fold selectivity, ${ }^{17}$ is mainly metabolized by the liver, and is excreted in feces. The aforementioned metabolites are excreted in the urine. ${ }^{18}$ The half-life of daprodustat (10 mg single dose) is about $1-2 \mathrm{hrs}$ in subjects with normal kidney function, ${ }^{19}$ whereas it is estimated to be 7 hrs in CKD patients (10 mg once daily). ${ }^{20}$ It is highly protein-bound (mainly albumin); therefore, it is not significantly eliminated by hemodialysis (HD). ${ }^{21}$ Indeed, in one Phase 1 study, pharmacokinetic parameters (area under the concentration-time curve, maximum observed concentration (Cmax), and time of occurrence of Cmax) were comparable between CKD stage 3-4, hemodialysis, and peritoneal dialysis patients. ${ }^{18}$ Daprodustat is mainly metabolized by CYP2CB; therefore, CYP2CB inhibitors may increase the plasma concentration of daprodustat when it is administrated with them. In contrast, CYP2CB inducers might reduce its plasma concentration. Taken together, the interview form of daprodustat recommends avoiding coadministration with CYP2CB inhibitors, including clopidogrel and trimethoprim, and CYP2CB inducers, such as rifampicin. ${ }^{16}$

\section{Therapeutic Clinical Trials}

\section{Phase 2 Studies}

\section{Non-Dialysis CKD Patients}

Holdstock et al conducted two randomized, blinded, controlled, phase 2a studies to assess hemoglobin doseresponse, safety, and tolerability of a 4-week administration of daprodustat. ${ }^{22}$ In this study, two patient groups were included: one was anemic CKD stage 3-5 patients group who were not dialysis-dependent and had not used rhEPO within the past 7 weeks (NCT01587898), and the other group was a hemodialysis patients group who had anemia and used stable doses of rhEPO (NCT01587924). In the non-dialysis group, 73 patients were randomized to receive placebo or $0.5 \mathrm{mg}, 2 \mathrm{mg}$, and $5 \mathrm{mg}$ daprodustat. In this study, daprodustat showed a dose-dependent increase in hemoglobin level, with a $5 \mathrm{mg}$ dose demonstrating an increase by $1.01 \mathrm{~g} / \mathrm{dl}$ (SD $0.26 \mathrm{~g} / \mathrm{dl}$ ) at 4 weeks after initiation, compared to placebo in which the change from the hemoglobin baseline was $-0.15 \mathrm{~g} /$ $\mathrm{dl}$ (SD $0.19 \mathrm{~g} / \mathrm{dl}$ ). In addition, plasma hepcidin and ferritin levels decreased, and total iron-binding capacity (TIBC) increased in the daprodustat group. On the other hand, plasma vascular endothelial growth factor (VEGF) levels were not significantly different across these treatment arms. In addition, the daprodustat group had decreased total cholesterol and low-density and high-density lipoprotein cholesterol levels in a dose-dependent manner (Table 1).

A higher daprodustat dose study was conducted wherein anemic non-hemodialysis-dependent CKD stage 3-5 patients $(\mathrm{N}=70)$ were randomized to placebo or $10 \mathrm{mg}, 25 \mathrm{mg}, 50 \mathrm{mg}$, and $100 \mathrm{mg}$ daprodustat at 4 weeks of administration (NCT01047397). ${ }^{20}$ These patients never underwent or discontinued ESA treatment for seven or more days or equivalent to the interval between

Table I Phase 2 Studies for Non-Hemodialysis-Dependent CKD Stage 3-5 Patients

\begin{tabular}{|c|c|c|c|c|c|c|c|c|c|}
\hline NCT Number & $\mathbf{n}$ & $\begin{array}{l}\text { Duration } \\
\text { (wk) }\end{array}$ & Dose (/Day) & Control & Ferritin & TIBC & Hepcidin & VEGF & Cholesterol \\
\hline NCTOI587898 22 & 73 & 4 & $0.5,2,5 \mathrm{mg}$ & Placebo & $\downarrow$ & $\uparrow$ & $\downarrow$ & $\rightarrow$ & $\downarrow$ \\
\hline NCTOI $047397^{20}$ & 70 & 4 & $10,25,50,100 \mathrm{mg}$ & Placebo & $\downarrow$ & $\uparrow$ & $\downarrow$ & Large variety & NA \\
\hline NCTOI $977573^{23}$ & 252 & 24 & $\begin{array}{l}\text { rhEPO-naïe: I, } 2,4 \mathrm{mg} \\
\text { rhEPO user: } 2 \mathrm{mg}\end{array}$ & rhEPO & $\downarrow$ & $\uparrow$ & $\downarrow$ & $\rightarrow$ & NA \\
\hline
\end{tabular}

Notes: Up-arrow indicates that the concentration increases. Down-arrow indicates that the concentration decreases. Right-arrow indicates that the concentration dose not change.

Abbreviations: CKD, chronic kidney disease; n, number of patients; wk, week; TIBC, total iron-binding capacity; VEGF, vascular endothelial growth factor; rhEPO, recombinant human erythropoietin; NA, not available. 
scheduled ESA doses. ${ }^{20}$ This study also demonstrated a dose-dependent increase of hemoglobin; however, 30\% of patients discontinued this study because their hemoglobin level increased by $>1 \mathrm{~g} / \mathrm{dl}$ in a 2 -week period or increased to $>13.5 \mathrm{~g} / \mathrm{dl}$, especially in patients who received $>25 \mathrm{mg}$ dose of daprodustat. Plasma hepcidin and ferritin levels decreased, and TIBC increased in all daprodustat doses. Highly variable changes from the baseline of VEGF level were noted. This study suggested a suitable daily dose of $<10 \mathrm{mg}$ daprodustat for future long-term clinical trials.

Holdstock et al conducted a large, 24-week, open-label, randomized, phase $2 \mathrm{~b}$ study, including anemic CKD stage 3-5 patients (NCT01977573), ${ }^{23}$ who were rhEPO-naïve (defined as non-rhEPO users within 8 weeks before screening) or rhEPO users (defined as receiving stable doses of rhEPO for 4 weeks before screening). In the rhEPO-naïve group, 180 patients were randomized $3: 1$, receiving $1 \mathrm{mg}$, $2 \mathrm{mg}$, or $4 \mathrm{mg}$ daprodustat based on their baseline hemoglobin level or standard care of rhEPO. In the rhEPO user group, 72 patients were randomized $1: 1$, receiving $2 \mathrm{mg}$ daprodustat or standard care of rhEPO. The daprodustat dose was unchanged for the first 4 weeks, after which the dose was adjusted to achieve or maintain the target hemoglobin level, which was different between Cohort 1 (9-10.5 $\mathrm{g} / \mathrm{dl}$ ) and Cohort $2(10-11.5 \mathrm{~g} / \mathrm{dl})$. At week 24, the mean hemoglobin level was $10.2 \mathrm{~g} / \mathrm{dl}$ in Cohort 1 and $10.9 \mathrm{~g} / \mathrm{dl}$ in Cohort 2, whereas it was $10.7 \mathrm{~g} / \mathrm{dl}$ (Cohort 1) and $11.0 \mathrm{~g} / \mathrm{dl}$ (Cohort 2) in the control rhEPO administration group. Between weeks 12 and $24,82 \%$ of patients maintained hemoglobin levels within the target range in the daprodustat group, and the rate was $66 \%$ in the control rhEPO administration group. In addition, the rate of patients who had $\geq 13 \mathrm{~g} / \mathrm{dl}$ hemoglobin over the course of 24 weeks was $4 \%$ (daprodustat) and 5\% (control) in the rhEPO-naïve group and $0 \%$ (daprodustat) and 3\% (control) in the rhEPO user group. The plasma EPO level was similar to the baseline in both the darpodustat and the rhEPO group. Similar to other phase 2 studies, plasma hepcidin and ferritin levels decreased, whereas the TIBC level increased, and the VEGF level did not change from the baseline. This study indicated that these doses of daprodustat could safely achieve target hemoglobin levels among rhEPO-naïve patients and maintain target hemoglobin levels among rhEPO users.

\section{Hemodialysis Patients}

Daprodustat was also effective in treating renal anemia among hemodialysis patients. In the abovementioned 4-week, randomized, phase $2 \mathrm{a}$ study, ${ }^{22}$ anemic hemodialysis-dependent patients who received a stable dose of rhEPO $(\mathrm{N}=83)$ were randomized, receiving $0.5 \mathrm{mg}$, $2 \mathrm{mg}$, and $5 \mathrm{mg}$ daprodustat or rhEPO treatment (NCT01587924). The mean hemoglobin level was maintained only in the $5 \mathrm{mg}$ daprodustat or rhEPO administration group, whereas it decreased in the $0.5 \mathrm{mg}$ and $2 \mathrm{mg}$ daprodustat group. EPO concentration did not accumulate over a 4-week study period in the daprodustat group, with about 12-fold higher median peak plasma EPO level in the rhEPO group than in any daprodustat dose group. Plasma hepcidin level did not increase from baseline in both $5 \mathrm{mg}$ daprodustat group and rhEPO group; however, it increased in $0.5 \mathrm{mg}$ and $2 \mathrm{mg}$ daprodustat groups. Similar to the results among non-hemodialysis-dependent CKD patients, no difference in the plasma VEGF level was noted between the daprodustat group and the rhEPO group (Table 2). This study showed that $0.5 \mathrm{mg}$ and $2 \mathrm{mg}$ daprodustat did not maintain the hemoglobin levels in hemodialysis patients when it was switched from standard rhEPO treatment.

Table 2 Phase 2 Studies for Hemodialysis Patients

\begin{tabular}{|l|l|l|l|l|l|l|l|l|l|}
\hline NCT Number & $\mathbf{n}$ & $\begin{array}{l}\text { Duration } \\
\text { (wk) }\end{array}$ & Dose & Control & Ferritin & TIBC & Hepcidin & VEGF & Cholesterol \\
\hline NCT01587924 22 & 83 & 4 & $0.5,2,5 \mathrm{mg} /$ day & rhEPO & $\downarrow(5 \mathrm{mg})$ & $\uparrow$ & $\rightarrow(5 \mathrm{mg})$ & $\rightarrow$ & $\downarrow$ \\
NCT01047397 & 37 & 4 & $10,25 \mathrm{mg} /$ day & Placebo & $\downarrow$ & $\uparrow$ & $\downarrow$ & Large variety & NA \\
NCT0201971924 & 97 & 4 & $4,6,8,10 \mathrm{mg} /$ day & Placebo & $\downarrow$ & $\uparrow$ & $\downarrow$ & $\rightarrow$ & $\downarrow$ \\
NCT02689206 & 103 & 29 days & $\begin{array}{l}10,15,25,30 \mathrm{mg} \text { thrice } \\
\text { a week }\end{array}$ & Placebo & $\downarrow$ & $\uparrow$ & $\downarrow$ & NA \\
NCT01977482 & 216 & 24 & $4,6,8,10,12 \mathrm{mg} /$ day & Placebo & $\downarrow$ & $\uparrow$ & $\downarrow$ & NA \\
\hline
\end{tabular}

Notes: Up-arrow indicates that the concentration increases. Down-arrow indicates that the concentration decreases. Right-arrow indicates that the concentration dose not change.

Abbreviations: $n$, number of patients; wk, week; TIBC, total iron-binding capacity; VEGF, vascular endothelial growth factor; rhEPO, recombinant human erythropoietin; NA, not available. 
A 4-week, randomized, phase 2a study included anemic hemodialysis patients who were ESA-naïve or discontinued ESA for $\geq 7$ days (NCT01047397). ${ }^{20}$ Patients ( $\mathrm{N}=$ 37) were randomized, receiving $10 \mathrm{mg}$ and $25 \mathrm{mg}$ daprodustat or placebo. Daprodustat was effective in increasing hemoglobin levels. However, $50 \%$ of patients stopped this study due to a high rate of hemoglobin increase. Based on this study, $<10 \mathrm{mg}$ dose of daprodustat was suggested to be suitable for the initial dose among hemodialysis patients, which is similar to non-hemodialysis-dependent CKD patients.

A 4-week, randomized, double-blind, phase 2 study was conducted to assess the efficacy and safety of daprodustat in Japanese hemodialysis patients (NCT02019719). ${ }^{24}$ Anemic hemodialysis patients who received ESA and discontinued ESA for 2-8 weeks prior to enrollment $(\mathrm{N}=97)$ were randomized, receiving $4 \mathrm{mg}, 6 \mathrm{mg}, 8 \mathrm{mg}$, and $10 \mathrm{mg}$ daprodustat or placebo if their hemoglobin level decreased by $\geq 0.5$ $\mathrm{g} / \mathrm{dl}$ and resulted in 8.5-10.5 $\mathrm{g} / \mathrm{dl}$ after ESA discontinuation. Daprodustat increased the hemoglobin level in a dosedependent manner. Also, a dose-dependent decrease of hepcidin and transferrin and increase of TIBC were observed in the daprodustat group. In addition, the cholesterol level decreased at week 4 in the daprodustat group, whereas the plasma VEGF level did not increase from baseline in both the daprodustat group and the placebo group. This study showed that daprodustat safely maintained and increased hemoglobin levels by $4-6 \mathrm{mg}$ and $8-10 \mathrm{mg}$ for Japanese hemodialysis patients, respectively.

Meadowcroft et al conducted a 24-week, randomized, open-label, phase $2 \mathrm{~b}$ study in which anemic hemodialysis patients who used stable doses of rhEPO for 8 weeks before randomization were included (NCT01977482). ${ }^{25}$ These patients $(\mathrm{N}=216)$ were randomized, receiving $4 \mathrm{mg}, 6 \mathrm{mg}, 8 \mathrm{mg}, 10 \mathrm{mg}$, or $12 \mathrm{mg}$ daprodustat or placebo, and received fixed doses of daprodustat or placebo for the first 4 weeks; after that, doses were adjusted or rhEPO was restarted to achieve or maintain the target hemoglobin range (10-11.5 mg/dl). At week 4, daprodustat increased hemoglobin levels in a dose-dependent manner, with changes evident from week $2 .^{25}$ At week 24 , $58 \%$ of patients reached the target hemoglobin range in either group, with a $6 \mathrm{mg}$ median daprodustat dose. Daprodustat was discontinued in $10 \%$ of patients due to a high hemoglobin level $(\geq 13 \mathrm{~g} / \mathrm{dl}), 82 \%$ of which occurred in patients who started $\geq 8 \mathrm{mg}$ daparodustat. Similar to other phase 2 studies, hepcidin and transferrin levels decreased, whereas TIBC increased, in the daprodustat group. No significant VEGF level differences were noted between the two groups. This study suggested that $<8 \mathrm{mg}$ initial dose of daprodustat was sufficient in reaching or maintaining the hemoglobin level within the target range among hemodialysis patients previously treated with rhEPO, in countries other than Japan. Also, a 29day, randomized, double-blind, placebo-controlled, phase 2 trial showed that thrice-a-week dosing of daprodustat $(10,15,25,30 \mathrm{mg})$ increased the hemoglobin level in a dose-dependent manner for anemic hemodialysis patients who were receiving a stable dose of rhEPO, switching it to daprodustat (NCT02689206), ${ }^{26}$ which suggests a potential for the drug to be applied using a thrice-weekly regimen.

\section{Phase 3 Studies}

Several phase 2 studies suggested suitable daprodustat doses, efficacy for renal anemia, and safety among nondialysis-dependent CKD and hemodialysis patients. To assess the efficacy and safety of daprodustat in a relatively larger number of patients for a long term, many phase 3 studies are underway, some of which were already completed. A 24-week, open-label, noncomparative, phase 3 study assessed the efficacy of daprodustat among Japanese hemodialysis patients with anemia who were not undergoing ESA therapy (NCT02829320) ${ }^{27}$ (Table 3). In this study, 28 patients received $4 \mathrm{mg}$ daprodustat once daily, with the dose being adjusted every 4 weeks according to a prespecified algorithm to achieve target hemoglobin level $(10-12 \mathrm{~g} / \mathrm{dl})$. At week 4 , the mean change of hemoglobin from baseline was $0.79 \mathrm{~g} / \mathrm{dl}$ (95\% CI, $0.53-1.05 \mathrm{~g} / \mathrm{dl})$. At week $24,82 \%$ of patients achieved the target hemoglobin level. During the 24-week study period, one participant had a hemoglobin level increase of $>2 \mathrm{~g} / \mathrm{dl}$ per 4 weeks, and three reached a high hemoglobin level $(>13 \mathrm{~g} / \mathrm{dl})$, the median daprodustat dose of which was $4 \mathrm{mg}$. Similar to phase 2 studies, ferritin and hepcidin levels decreased, whereas TIBC level increased. This study showed that daprodustat was effective for renal anemia treatment among Japanese hemodialysis patients who were not receiving ESA treatment and that the initial dose of $4 \mathrm{mg} /$ day was sufficient and safe to achieve the target hemoglobin level.

Akizawa et al conducted a 52-week, randomized, double-blind, phase 3 study to assess the noninferiority of daprodustat in maintaining the hemoglobin level among Japanese hemodialysis patients and its safety compared to darbepoetin alfa (NCT02969655). ${ }^{28}$ Every participant received a stable dose of ESA treatment (darbepoetin 
Table 3 Phase 3 Studies for Hemodialysis and Non-Dialysis Patients

\begin{tabular}{|c|c|c|c|c|c|c|}
\hline NCT Number (Study Name) & Enrollment & Pt Type & $\begin{array}{l}\text { Dose } \\
\text { Frequency }\end{array}$ & Control & Status & Location \\
\hline NCT0282932027 & 28 & Hemodialysis & Once a day & Non & Completed & Japan \\
\hline NCT02969655 ${ }^{28}$ & 271 & Hemodialysis & Once a day & Darbepoetin alfa & Completed & Japan \\
\hline NСT02791763 ${ }^{16}$ & 355 & $\begin{array}{l}\text { CKD, peritoneal } \\
\text { dialysis }\end{array}$ & Once a day & $\begin{array}{l}\text { Epoetin beta } \\
\text { pegol }\end{array}$ & Completed & Japan \\
\hline NCT03029208 (ASCEND-ID) & 330 & Incident dialysis & Once a day & Darbepoetin alfa & $\begin{array}{l}\text { Active (not } \\
\text { recruiting) }\end{array}$ & International \\
\hline NCT03400033 (ASCEND-TD) & 407 & Hemodialysis & Thrice a week & $\begin{array}{l}\text { Epoetin alpha } \\
\text { Placebo }\end{array}$ & Completed & International \\
\hline NCT03409I07 (ASCEND-NHQ) & 600 & CKD & Once a day & Placebo & $\begin{array}{l}\text { Active (not } \\
\text { recruiting) }\end{array}$ & International \\
\hline NCT02879305 (ASCEND-D) & 2964 & Hemodialysis & Once a day & rhEPO & $\begin{array}{l}\text { Active (not } \\
\text { recruiting) }\end{array}$ & International \\
\hline NCT02876835 (ASCEND-ND) & 4500 & CKD & Once a day & Darbepoetin alfa & $\begin{array}{l}\text { Active (not } \\
\text { recruiting) }\end{array}$ & International \\
\hline
\end{tabular}

Abbreviations: Pt type, patient type; CKD, chronic kidney disease; rhEPO, recombinant human erythropoietin.

alfa $10-60 \mu \mathrm{g} /$ week, epoetin (including biosimilars) $\leq 9000$ IU/week, or epoetin beta pegol up to $250 \mu \mathrm{g} / 4$ weeks) at least 10 weeks before screening. Among 271 patients, 136 were randomized, receiving $4 \mathrm{mg}$ daprdodustat with dose titration every 4 weeks based on a prespecified algorithm to achieve or maintain the target hemoglobin level (10-12 $\mathrm{g} / \mathrm{dl}$ ). During weeks 40-52, the mean hemoglobin level was $10.9 \mathrm{~g} / \mathrm{dl}$ and $10.8 \mathrm{~g} / \mathrm{dl}$ in the daprodustat group and darbepoetin alfa group, respectively. This study showed that daprodustat was noninferior to darbepoetin alfa because the lower limit of $95 \% \mathrm{CI}$ for the treatment difference between the two groups exceeded the predefined noninferiority margin of $-1.0 \mathrm{~g} / \mathrm{dl}(0.1 \mathrm{~g} / \mathrm{dl}, 95 \% \mathrm{CI}$ -0.1 to $0.2 \mathrm{~g} / \mathrm{dl}$ ). In the daprodustat group, $88 \%$ of patients achieved the target hemoglobin level during weeks $40-52$, and $90 \%$ achieved that in the darbepoetin alfa group. In addition, the proportion of patients with a $>2 \mathrm{~g} / \mathrm{dl}$ hemoglobin level increase over any 4 weeks and $>13 \mathrm{~g} / \mathrm{dl}$ hemoglobin level was similar between the daprodustat group and the darbepoetin alfa group ( $<1 \%$ vs $1 \%, 5 \%$ vs $6 \%$, respectively). Both groups had a decreased hepcidin level, and the daprodustat group had a larger percent change from baseline at week 52 than the darbepoetin alfa group ( $-37 \%$ vs $-20 \%)$. Also, both groups had a decreased ferritin level. TIBC and serum iron level increased in the daprodustat group, which did not change from baseline in the darbepoetin alfa group. In this study, supplemental iron therapy, including intravenous (IV) and oral, was allowed to be initiated from week 4 as needed. And the proportion of patients who were started with IV iron was lower in the daprodustat group than in the darbepoetin alfa group, and oral iron was similarly used in both groups. According to this study, the starting dose of $4 \mathrm{mg}$ daprodustat, with a predefined dose titration algorithm, was noninferior to darbepoetin alfa because it achieved the target hemoglobin range without causing a rapid increase or overshooting of hemoglobin when switched from darbepoetin alfa, also suggesting that daprodustat might promote iron absorption and improve iron utilization compared to darbepoetin alfa.

In Japan, a 52-week, randomized, open-label, phase 3 study was conducted to assess the noninferiority of daprodustat in achieving or maintaining the target hemoglobin level (11-13 g/dl) among Japanese non-hemodialysisdependent CKD patients $(\mathrm{N}=299)$ who were ESA-naïve or ESA users, compared to epoetin beta pegol (NCT02791763). ${ }^{29}$ For ESA-naïve, non-hemodialysisdependent CKD patients, the initial dose of daprodustat was either $2 \mathrm{mg}$ or $4 \mathrm{mg}$ based on their baseline hemoglobin level, with a prespecified dose titration algorithm. The initial dose of daprodustat was $4 \mathrm{mg}$, which was titrated following the algorithm in non-hemodialysis-dependent CKD patients previously treated with ESA. During 
weeks $40-52$, the mean hemoglobin level in the daprodustat group was noninferior to the epoetin beta pegol group, as the difference in the lower limit of $95 \%$ CI between the treatment groups exceeded the predefined noninferiority margin of $-1.0 \mathrm{~g} / \mathrm{dl}(0.1 \mathrm{~g} / \mathrm{dl}, 95 \% \mathrm{CI}-0.07$ to $0.28 \mathrm{~g} /$ dl). Also, in this study, the efficacy of daprodustat was evaluated among peritoneal dialysis patients $(\mathrm{N}=56)$. The mean hemoglobin level at weeks 40-52 was $12.09 \mathrm{~g} / \mathrm{dl}$, achieving the target hemoglobin level (11-13 g/dl) in patients who received daprodustat. This study showed daprodustat was also effective in achieving and maintaining the target hemoglobin level among Japanese nonhemodialysis-dependent CKD and peritoneal dialysis patients.

As other international large phase 3 studies are ongoing or completed, these results are warranted to confirm the efficacy of daprodustat and recognize its safety profile more precisely in several groups of patients, including hemodialysis, hemodialysis-independent CKD, and incident dialysis (Table 3).

\section{Adverse Effects}

As HIF $1 \alpha$ and HIF $2 \alpha$ regulate the expression of various genes, HIF stabilization consequently has several effects beyond erythropoietin production, like angiogenesis, cell survival, and energy metabolism. ${ }^{12}$ For example, von Hippel Lindau disease has a germline mutation in VHL gene that results in VHL protein dysfunction and HIF stabilization, and patients with this disease are at high risk for several diseases, including clear cell renal cell carcinoma, pheochromocytoma, and retinal and cerebral hemangioblastoma. ${ }^{30}$ Also, patients with Chuvash polycythemia, which is caused by a specific mutation in VHL gene, have a higher baseline pulmonary artery pressure. ${ }^{31}$ Based on these facts, HIF activation by systemic HIF-PH inhibitor administration could promote cancer progression, pulmonary hypertension, thromboembolic complications, and retinopathy.

Contrary to these concerns, daprodustat was welltolerated in phase 3 studies. In Japanese dialysis patients who were not undergoing ESA treatment, the death, cancer, myocardial infarction, or stroke incidence was not observed during the 24-week study period. ${ }^{27}$ Nasopharyngitis was the most common adverse event (32\%), which occurred in $\geq 2$ patients. Cholesterol decreased during this study. Shunt occlusion, which is a serious adverse event, was reported in two patients (7\%). Ophthalmologic assessments were regularly conducted during this study period, with only one patient considered to develop an ocular adverse event. Among Japanese hemodialysis patients who received ESA treatment, death and cancer progression were not reported during the 52 -week study period. ${ }^{28}$ Among the adverse events reported in $\geq 5 \%$ of patients per group, contusion and diarrhea were reported $\geq 5 \%$ more frequently in the daprodustat group than in the darbepoetin alfa group. Similar to a phase 2 study, ${ }^{25}$ the incidence of hyperkalemia was reported in the daprodustat group (3\%) compared to the darbepoetin alfa group (1\%). However, both groups showed no clinically significant change from baseline in potassium level. Each group similarly reported hypertension, and the rate of antihypertensive medication change because of increased blood pressure was $38 \%$ and $49 \%$ in the daprodustat and darbepoetin alfa groups. The incidence rate of shunt occlusion and shunt stenosis had no difference between the daprodustat and darbepoetin alfa groups ( $<1 \%$ vs $2 \%, 3 \%$ vs $4 \%$, respectively). Regular ophthalmologic evaluation was performed in this study, and the rate of ocular adverse event incidence was similar between the two groups. In Japanese non-hemodialysis-dependent CKD patients, the rate of treatment-related adverse events was $6 \%$, including retinal hemorrhage $(<1 \%)$ in the daprodustat group. ${ }^{29}$ Among Japanese peritoneal dialysis patients, treatment-related adverse events were reported in $14 \%$ of the patients, with each occurrence rate of pulmonary embolism, pulmonary artery hypertension, and retinal hemorrhage being $2 \%{ }^{29}$

Theoretically, HIF stabilization using daprodustat was supposed to increase plasma VEGF level and result in ocular adverse events, including retinal neovascularization and retinal hemorrhage. However, plasma VEGF level increased only when a high dose of daprodustat $(50-100 \mathrm{mg})$ was administered to healthy participants, ${ }^{19}$ and several phase 2 studies showed that daprodustat had no effect on circulating VEGF levels. ${ }^{22-25}$ In addition, considering that ocular adverse events did not increase in phase 3 studies, ${ }^{27,28}$ the therapeutic dose of daprodustat would not increase plasma VEGF levels.

According to prior studies, daprodustat was safely used to treat renal anemia in both hemodialysis and nonhemodialysis-dependent CKD patients. However, large, long-term studies are required for the precise evaluation of cardiovascular risk. Two large phase 3 studies are ongoing to examine the efficacy and the time to the first occurrence of major adverse cardiovascular events among hemodialysis patients (ASCEND-D trial) and non- 
hemodialysis-dependent CKD patients (ASCEND-ND trial) (Table 3). Although the incidence of hyperkalemia increased in two phase 3 trials of roxadustat, ${ }^{14,15}$ it had no significant increase in phase 3 studies that evaluate daprodustat efficacy for renal anemia among hemodialysis and non-hemodialysis-dependent CKD patients. ${ }^{28,29}$ To confirm whether adverse event profile differences exist among several HIF-PH inhibitors, more detailed phase 3 or phase 4 studies of each HIF-PH inhibitor should be required.

\section{Other HIF-PH Inhibitors}

Additional HIF-PH inhibitors, such as roxadustat, vadadustat, and enarodustat, were also approved in Japan for the treatment of renal anemia among hemodialysis and nonhemodialysis-dependent CKD patients. All HIF-PH inhibitors showed improvement of renal anemia. Also, Haase et al summarized the effect of HIF-PH inhibitors regarding iron metabolism and the effect on iron utilization was considered as a class effect of HIF-PH inhibitors. ${ }^{32}$ However, several effects turned out to be different among HIF-PH inhibitors. Cholesterol-lowering effect was reported in roxadustat ${ }^{14}$ and daprodustat. On the other hand, other HIF-PH inhibitors did not show the cholesterol-lowering effect. A phase $2 \mathrm{~b}$ study that assessed the effect of vadadustat among non-dialysis-dependent CKD patients reported that the mean change from baseline in cystatin $\mathrm{C}$ levels was significantly small in the vadadustat group (34.9 $\mathrm{ng} / \mathrm{mL})$ compared to the placebo group $(298.2 \mathrm{ng} / \mathrm{mL}){ }^{33}$ In addition, the pharmacological properties were different among HIF-PH inhibitors. ${ }^{34}$ The difference in potency to stabilize HIF suggested that the speed with which hemoglobin levels increase might be variable. ${ }^{35}$ Moreover, whether the safety profile is different among each HIF-PH inhibitor due to their structural difference or distinct metabolic profiles remains unclear. ${ }^{36}$ Therefore, more clinical studies of each HIF-PH inhibitor are needed, and these results should be compared with each other in the future.

\section{Possible Pleiotropic Effects of HIF-PH Inhibitors Beyond Renal Anemia Therapy}

Several preclinical studies showed several beneficial effects of HIF-PH inhibitors other than renal anemia improvement. $^{12}$ A study using enarodustat, one of the HIF-PH inhibitors, reported that plasma adiponectin level was maintained and enarodustat might have renoprotective effects in obese type 2 diabetes mouse model ${ }^{37}$ and obese mice with a high-fat diet. ${ }^{38}$ Another study suggested that enarodustat reduced cardiac hypertrophy and myocardial fibrosis in the 5/6 nephrectomy rodent model with the nitric oxide synthase inhibitor. ${ }^{39}$ On the other hand, some preclinical studies showed that HIF-PH inhibitors had harmful effects. In the murine severe polycystic kidney disease model, the HIF-PH inhibitor enhanced cyst growth through HIF1 $\alpha$ activation. ${ }^{40}$ Also, one in vitro study demonstrated that roxadustat promoted phosphateinduced vascular smooth muscle cell calcification. ${ }^{41}$ Therefore, post-marketing surveillance is also required in assessing the safety concerns, because of the strict limitations of clinical trials for participants based on their inclusion and exclusion criteria; meanwhile, numerous CKD patients who have several comorbidities might receive HIF-PH inhibitors in the clinical practice setting. Future studies are required to assess efficacy for renal anemia, safety, and other potential benefits of daprodustat. ${ }^{42}$

\section{Summary}

Several phase 3 and phase 2 studies showed daprodustat to be effective in improving or maintaining hemoglobin levels among hemodialysis and non-hemodialysisdependent CKD patients, especially the Japanese ones. And this effect was noninferior to ESA treatment, with iron utilization improvement. Although there was a concern that several adverse effects might happen due to HIF stabilization through systemic PHD inhibition, adverse events of particular interest, such as cancer, hypertension, and shunt occlusion, did not increase in the daprodustat group during the study period of up to 1 year, compared to the ESA treatment group. Also, no clinically significant increase in plasma VEGF level was noted, and no ocular events were observed. Based on these results, daprodustat was approved in Japan for the treatment of renal anemia among hemodialysis and non-hemodialysisdependent CKD patients in June 2020. ${ }^{16}$ International phase 3 clinical trials for daprodustat are underway to evaluate the efficacy and safety in depth.

Daprodustat was expected to possibly not be linked to an increase in cardiovascular disease risk because phase 2 studies showed that daprodustat could increase the hemoglobin level without excessive EPO production and plasma EPO level was lower in the daprodustat than in the rhEPO group in hemodialysis patients. $^{22}$ To evaluate whether daprodustat affects cardiovascular events as a coprimary 
endpoint, ASCEND-D and ASCEND-ND trials are ongoing. In addition, other potentially beneficial aspects of daprodustat are being assessed. In ASCEND-NHQ trial, which is an international, randomized, double-blind, phase 3 study and includes non-hemodialysis-dependent CKD patients, health-related quality of life is evaluated as one of the secondary endpoints.

\section{Conclusion}

Daprodustat showed efficacy for renal anemia in both hemodialysis- and non-hemodialysis-dependent CKD patients and it was approved for the treatment of renal anemia in Japan. If the international phase 3 studies reveal positive results, daprodustat may also be another valuable treatment for renal anemia in many countries other than Japan.

\section{Disclosure}

M.N. has received research grant from JT, and Kyowa Kirin Co. Ltd., and personal fees from GlaxoSmithKline, Astellas, Mitsubishi Tanabe Pharma Corporation, Bayer Yakuhin Ltd., Torii Pharmaceutical Co. Ltd, and AstraZeneca, outside the submitted work. T.T. has received research grant from JT and Bayer. T.I. declared no conflicts of interest. The authors report no other conflicts of interest in this work.

\section{References}

1. Webster AC, Nagler EV, Morton RL, Masson P. Chronic kidney disease. Lancet. 2017;389(10075):1238-1252. doi:10.1016/S01406736(16)32064-5

2. Koury MJ, Haase VH. Anaemia in kidney disease: harnessing hypoxia responses for therapy. Nat Rev Nephrol. 2015;11(7):394-410.

3. Weiner DE, Tighiouart H, Vlagopoulos PT, et al. Effects of anemia and left ventricular hypertrophy on cardiovascular disease in patients with chronic kidney disease. J Am Soc Nephrol. 2005;16(6):1803-1810. doi:10.1681/ASN.2004070597

4. Drueke TB, Locatelli F, Clyne N, et al. Normalization of hemoglobin level in patients with chronic kidney disease and anemia. $N$ Engl J Med. 2006;355(20):2071-2084. doi:10.1056/NEJMoa062276

5. Finkelstein FO, Story K, Firanek C, et al. Health-related quality of life and hemoglobin levels in chronic kidney disease patients. Clin $\mathrm{J} \mathrm{Am}$ Soc Nephrol. 2009;4(1):33-38. doi:10.2215/CJN.00630208

6. Lewis EF, Pfeffer MA, Feng A, et al. Darbepoetin alfa impact on health status in diabetes patients with kidney disease: a randomized trial. Clin J Am Soc Nephrol. 2011;6(4):845-855. doi:10.2215/ CJN.06450710

7. Besarab A, Bolton WK, Browne JK, et al. The effects of normal as compared with low hematocrit values in patients with cardiac disease who are receiving hemodialysis and epoetin. N Engl J Med. 1998;339 (9):584-590. doi:10.1056/NEJM199808273390903

8. Pfeffer MA, Burdmann EA, Chen CY, et al. A trial of darbepoetin alfa in type 2 diabetes and chronic kidney disease. $N$ Engl J Med. 2009;361 (21):2019-2032. doi:10.1056/NEJMoa0907845
9. Singh AK, Szczech L, Tang KL, et al. Correction of anemia with epoetin alfa in chronic kidney disease. $N$ Engl J Med. 2006;355 (20):2085-2098. doi:10.1056/NEJMoa065485

10. Koulouridis I, Alfayez M, Trikalinos TA, Balk EM, Jaber BL. Dose of erythropoiesis-stimulating agents and adverse outcomes in CKD: a metaregression analysis. Am J Kidney Dis. 2013;61(1):44-56. doi:10.1053/j.ajkd.2012.07.014

11. Kanbay M, Perazella MA, Kasapoglu B, Koroglu M, Covic A. Erythropoiesis stimulatory agent-resistant anemia in dialysis patients: review of causes and management. Blood Purif. 2010;29(1):1-12. doi:10.1159/000245041

12. Maxwell PH, Eckardt KU. HIF prolyl hydroxylase inhibitors for the treatment of renal anaemia and beyond. Nat Rev Nephrol. 2016;12 (3):157-168. doi:10.1038/nrneph.2015.193

13. Haase VH. Regulation of erythropoiesis by hypoxia-inducible factors. Blood Rev. 2013;27(1):41-53. doi:10.1016/j.blre.2012.12.003

14. Chen N, Hao C, Liu BC, et al. Roxadustat treatment for anemia in patients undergoing long-term dialysis. $N$ Engl J Med. 2019;381 (11):1011-1022. doi:10.1056/NEJMoa1901713

15. Chen N, Hao C, Peng X, et al. Roxadustat for anemia in patients with kidney disease not receiving dialysis. $N$ Engl J Med. 2019;381 (11):1001-1010. doi:10.1056/NEJMoa1813599

16. GlaxoSmithKline. Duvroq (Daprodustat): Japanese prescribing information; 2020. Available from: https://www.pmda.go.jp/ PmdaSearch/iyakuDetail/ResultDataSetPDF/340278_ 3999049F1027_1_01\#page=4. Accessed October 15, 2020.

17. Ariazi JL, Duffy KJ, Adams DF, et al. Discovery and preclinical characterization of GSK1278863 (Daprodustat), a small molecule hypoxia inducible factor-prolyl hydroxylase Inhibitor for Anemia. J Pharmacol Exp Ther. 2017;363(3):336-347. doi:10.1124/jpet.117.242503

18. Caltabiano S, Cizman B, Burns O, et al. Effect of renal function and dialysis modality on daprodustat and predominant metabolite exposure. Clin Kidney J. 2019;12(5):693-701. doi:10.1093/ckj/sfz013

19. Hara K, Takahashi N, Wakamatsu A, Caltabiano S. Pharmacokinetics, pharmacodynamics and safety of single, oral doses of GSK1278863, a novel HIF-prolyl hydroxylase inhibitor, in healthy Japanese and Caucasian subjects. Drug Metab Pharmacokinet. 2015;30(6):410-418. doi:10.1016/j.dmpk.2015.08.004

20. Brigandi RA, Johnson B, Oei C, et al. A novel hypoxia-inducible factor-prolyl hydroxylase inhibitor (GSK1278863) for Anemia in CKD: a 28-day, phase 2A randomized trial. Am J Kidney Dis. 2016;67(6):861-871. doi:10.1053/j.ajkd.2015.11.021

21. Sanghani NS, Haase VH. Hypoxia-inducible factor activators in renal anemia: current clinical experience. Adv Chronic Kidney Dis. 2019;26(4):253-266. doi:10.1053/j.ackd.2019.04.004

22. Holdstock L, Meadowcroft AM, Maier R, et al. Four-week studies of oral hypoxia-inducible factor-prolyl hydroxylase inhibitor GSK1278863 for treatment of Anemia. J Am Soc Nephrol. 2016;27 (4):1234-1244. doi:10.1681/ASN.2014111139

23. Holdstock L, Cizman B, Meadowcroft AM, et al. Daprodustat for anemia: a 24-week, open-label, randomized controlled trial in participants with chronic kidney disease. Clin Kidney J. 2019;12 (1):129-138. doi:10.1093/ckj/sfy013

24. Akizawa T, Tsubakihara Y, Nangaku M, et al. Effects of daprodustat, a novel hypoxia-inducible factor prolyl hydroxylase inhibitor on anemia management in Japanese hemodialysis subjects. Am $J$ Nephrol. 2017;45(2):127-135. doi:10.1159/000454818

25. Meadowcroft AM, Cizman B, Holdstock L, et al. Daprodustat for anemia: a 24-week, open-label, randomized controlled trial in participants on hemodialysis. Clin Kidney J. 2019;12(1):139-148. doi:10.1093/ckj/sfy014

26. Bailey CK, Caltabiano S, Cobitz AR, Huang C, Mahar KM, Patel VV. A randomized, 29-day, dose-ranging, efficacy and safety study of daprodustat, administered three times weekly in patients with anemia on hemodialysis. BMC Nephrol. 2019;20(1):372. doi:10.1186/s12882-019-1547-z 
27. Tsubakihara Y, Akizawa T, Nangaku M, et al. A 24-Week Anemia correction study of daprodustat in Japanese dialysis patients. Ther Apher Dial. 2020;24(2):108-114. doi:10.1111/1744-9987.12962

28. Akizawa T, Nangaku M, Yonekawa T, et al. Efficacy and safety of daprodustat compared with darbepoetin alfa in Japanese hemodialysis patients with anemia: a randomized, double-blind, phase 3 Trial. Clin J Am Soc Nephrol. 2020;15(8):1155-1165. doi:10.2215/CJN.1601 1219

29. angaku M, Hamano T, Akizawa T, et al. Daprodustat Compared with Epoetin Beta Pegol for Anemia in Japanese Patients Not on Dialysis: A 52-Week Randomized Open-Label Phase 3 Trial. Am J Nephrol. 2021;9:1-10. doi: 10.1159/000513103.

30. Gossage L, Eisen T, Maher ER. VHL, the story of a tumour suppressor gene. Nat Rev Cancer. 2015;15(1):55-64. doi:10.1038/nrc3844

31. Smith TG, Brooks JT, Balanos GM, et al. Mutation of von Hippel-Lindau tumour suppressor and human cardiopulmonary physiology. PLoS Med. 2006;3(7):e290. doi:10.1371/journal.pmed. 0030290

32. Haase VH. HIF-prolyl hydroxylases as therapeutic targets in erythropoiesis and iron metabolism. Hemodial Int. 2017;21(Suppl 1):S110S124. doi:10.1111/hdi.12567

33. Pergola PE, Spinowitz BS, Hartman CS, Maroni BJ, Haase VH. Vadadustat, a novel oral HIF stabilizer, provides effective anemia treatment in nondialysis-dependent chronic kidney disease. Kidney Int. 2016;90(5):1115-1122. doi:10.1016/j.kint.2016.07.019

34. Yeh TL, Leissing TM, Abboud MI, et al. Molecular and cellular mechanisms of HIF prolyl hydroxylase inhibitors in clinical trials. Chem Sci. 2017;8(11):7651-7668. doi:10.1039/C7SC02103H

35. Kuriyama S, Maruyama Y, Honda H. A new insight into the treatment of renal anemia with HIF stabilizer. Renal Replacement Therapy. 2020;6:1. doi:10.1186/s41100-020-00311-x
36. Sugahara M, Tanaka T, Nangaku M. Prolyl hydroxylase domain inhibitors as a novel therapeutic approach against anemia in chronic kidney disease. Kidney Int. 2017;92(2):306-312. doi:10.1016/j. kint.2017.02.035

37. Sugahara M, Tanaka S, Tanaka T, et al. Prolyl hydroxylase domain inhibitor protects against metabolic disorders and associated kidney disease in obese type 2 diabetic mice. J Am Soc Nephrol. 2020;31 (3):560-577. doi:10.1681/ASN.2019060582

38. Saito H, Tanaka T, Sugahara M, et al. Inhibition of prolyl hydroxylase domain (PHD) by JTZ-951 reduces obesity-related diseases in the liver, white adipose tissue, and kidney in mice with a high-fat diet. Lab Invest. 2019;99(8):1217-1232. doi:10.1038/s41374-0190239-4

39. Uchida L, Tanaka T, Saito H, et al. Effects of a prolyl hydroxylase inhibitor on kidney and cardiovascular complications in a rat model of chronic kidney disease. Am J Physiol Renal Physiol. 2020;318(2): F388-F401. doi:10.1152/ajprenal.00419.2019

40. Kraus A, Peters DJM, Klanke B, et al. HIF-1alpha promotes cyst progression in a mouse model of autosomal dominant polycystic kidney disease. Kidney Int. 2018;94(5):887-899. doi:10.1016/j. kint.2018.06.008

41. Mokas S, Lariviere R, Lamalice L, et al. Hypoxia-inducible factor-1 plays a role in phosphate-induced vascular smooth muscle cell calcification. Kidney Int. 2016;90(3):598-609. doi:10.1016/j. kint.2016.05.020

42. Yap DYH, McMahon L, Hao C-M, et al. Recommendations on the proper use of HIF-PH inhibitors by the Asia-Pacific Society of Nephrology (APSN). Nephrology.
Therapeutics and Clinical Risk Management

\section{Publish your work in this journal}

Therapeutics and Clinical Risk Management is an international, peerreviewed journal of clinical therapeutics and risk management, focusing on concise rapid reporting of clinical studies in all therapeutic areas, outcomes, safety, and programs for the effective, safe, and sustained use of medicines. This journal is indexed on PubMed Central, CAS,

\section{Dovepress}

EMBase, Scopus and the Elsevier Bibliographic databases. The manuscript management system is completely online and includes a very quick and fair peer-review system, which is all easy to use. Visit http://www.dovepress.com/testimonials.php to read real quotes from published authors. 